\title{
Temperature-dependent vacuum tunneling spectroscopy of rare-earth manganates showing colossal magnetoresistance and charge ordering
}

\author{
A. Biswas ${ }^{1, *}$, A.K. Raychaudhuri ${ }^{1, * *}$, A. Arulraj $^{2}$, C.N.R. Rao ${ }^{2}$ \\ ${ }^{1}$ Department of Physics, Indian Institute of Science, Bangalore 560 012, India \\ ${ }^{2}$ Solid State and Structural Chemistry Unit, Indian Institute of Science, Bangalore 560012 , India \\ Received: 25 July 1997/Accepted: 1 October 1997
}

\begin{abstract}
We report here the results of vacuum tunneling spectroscopy of rare-earth manganates which show colossal magnetoresistance (CMR) and charge ordering (CO). Three samples with different hole concentrations and average A site cation radii have been studied. A charge-ordering gap $\left(\Delta_{\mathrm{CO}}\right)$ opens up in the density of states (DOS) near $E_{\mathrm{F}}$, below the charge-ordering transition. A gap comparable to the transport gap opens up in the paramagnetic state also. There is an absence of gap only in the ferromagnetic metallic state. We have measured the temperature dependence of these quantities.
\end{abstract}

Since the recent discovery of colossal magnetoresistance (CMR) in hole-doped rare-earth manganates, their novel transport properties have been investigated extensively [1, 2]. These rare-earth manganates with general formula $\mathrm{RE}_{(1-x)} \mathrm{A}_{x} \mathrm{MnO}_{3}(\mathrm{RE}=\mathrm{La}, \mathrm{Nd}, \mathrm{Pr}, \mathrm{Sm}$, etc. and $\mathrm{A}=\mathrm{Ca}, \mathrm{Sr}$, $\mathrm{Ba}, \mathrm{Pb}$, etc.) show a fine interplay of magnetic, Coulomb and lattice interactions which manifest themselves through such phenomena as insulator-metal transitions, CMR and charge ordering (CO). Mn ions in these compounds have mixed valency with the $\mathrm{Mn}^{4+} / \mathrm{Mn}^{3+}$ ratio $\approx x /(1-x)$, depending on the exact value of the oxygen stoichiometry. These oxides belong to the $\mathrm{ABO}_{3}$ perovskite class of structure where the A site is occupied by the $\operatorname{Re}$ or $\mathrm{M}$ ions and the $\mathrm{B}$ site by the Mn ions. For certain values of $x \approx 0.2-0.3$ these materials show CMR close to the ferromagnetic transition temperature, $T_{\mathrm{C}}$, when fields of a few tesla are applied. The stability of the ferromagnetic metallic state depends on the bandwidth $W$ which can be controlled through external pressure [3] or alternatively through the average radius of an A-site cation $\left(\left\langle r_{A}\right\rangle\right)$ [4]. As $\left\langle r_{A}\right\rangle$ becomes smaller the lattice is distorted leading to a smaller transfer matrix and a smaller $W$. This in turn reduces the $T_{\mathrm{C}}$. When $\left\langle r_{A}\right\rangle$ is sufficiently small the ferromagnetic $T_{\mathrm{C}}$ vanishes, as in solids with $\mathrm{RE}=\mathrm{Nd}$, Pr and $\mathrm{A}=\mathrm{Ca}$ [4]. Lattice effects play an important role in both carrier transport and magnetism in these materials by con-

\footnotetext{
* E-mail: amlan@physics.iisc.ernet.in

** E-mail: arup@physics.iisc.ernet.in
}

trolling the bandwidth and the Jahn-Teller (J-T) distortion around the $\mathrm{Mn}^{3+}$ ions, which results in strong electron-lattice interaction.

For materials with smaller A-site cations $\left(\left\langle r_{A}\right\rangle \leq 1.25 \AA\right)$ near half-filling ( $x \approx 1 / 2)$, the Coulomb interaction can overcome the kinetic energy of the electrons leading to arrangement of the $\mathrm{Mn}^{3+}$ and $\mathrm{Mn}^{4+}$ in alternate lattice sites. This $\mathrm{CO}$ transition is associated with large lattice distortions and leads to localization of the carrier and the material becomes insulating below the transition temperature, $T_{\mathrm{CO}}$ [5]. For materials like $\mathrm{Nd}_{0.5} \mathrm{Sr}_{0.5} \mathrm{MnO}_{3}\left(\left\langle r_{A}\right\rangle \approx 1.22 \AA\right)$ the $\mathrm{CO}$ transition is associated with the onset of antiferromagnetic ordering i.e. $T_{\mathrm{CO}} \approx T_{\mathrm{N}}$, the Néel temperature [5]. In materials with even smaller $\left\langle r_{A}\right\rangle(\leq 1.18 \AA)$ the $\mathrm{CO}$ transition is more gradual and the AFM ordering may take place at a lower temperature $[6,7]$. Lattice distortion (through the J-T effect) plays an important role in ushering in the $\mathrm{CO}$ insulating state. The orthorhombic lattice distortion generally arises as a contraction of the $c$-axis and a relative expansion of the $a$-and $b$-axes of the unit cell [6-8]. On cooling, the distortion generally increases, stabilizing the $\mathrm{CO}$ state as the ground state. However, the $\mathrm{CO}$ state can become unstable and collapse in the presence of a strong magnetic field [5].

From the above discussion it is clear that there is a need for a careful measurement of the density of states (DOS) at the Fermi level $E_{\mathrm{F}}$ in these materials as a function of temperature in order to see how the DOS at $E_{\mathrm{F}}$ varies at the different transition temperatures. In particular, one would like to know whether there is a gap at the Fermi level at the CO transition. With this specific aim we have investigated the DOS near the $E_{\mathrm{F}}$ in certain selected samples of these materials by using the technique of vacuum tunneling using a variable temperature scanning tunneling microscope (STM). We present data on three materials namely $\mathrm{La}_{0.8} \mathrm{Ca}_{0.2} \mathrm{MnO}_{3}$ (sample A), $\mathrm{Nd}_{0.5} \mathrm{Sr}_{0.5} \mathrm{MnO}_{3}$ (sample B) and (NdLa) ${ }_{0.5} \mathrm{Ca}_{0.5} \mathrm{MnO}_{3}$ (sample C). Sample A shows CMR behavior and no CO transition. Sample B shows a ferromagnetic metallic state followed by transition to a low-temperature CO state. Sample $\mathrm{C}$ shows an incipient $\mathrm{CO}$ state which collapses into a metallic ferromagnetic state on cooling. Our tunneling spectroscopy study has clearly brought out the different transitions as seen in the DOS. 


\section{Experiment}

The polycrystalline materials used in this work were prepared by the usual solid-state method. The samples were characterized by X-rays, and titration methods were used to fix the exact $\mathrm{Mn}^{4+}$ concentration. The details of sample preparation and characterization are given elsewhere $[9,10]$. The electrical resistivity $(\rho)$ was determined for $500 \mathrm{~K}>T>4.2 \mathrm{~K}$ using the usual four-probe technique.

The tunneling spectroscopy (TS) investigation was carried out with a custom-made low-temperature STM using a platinum-rhodium tip $[11,12]$. The schematic diagram of the STM electronics is given in Fig. 1. The sample was kept on a temperature-controlled copper block. The thermometer on the sample holder and the thermometer used for the resistivity measurements are both calibrated with the same standard thermometers. While recording the $I-V$ spectra, the temperature was stable within an accuracy of $10 \mathrm{mK}$. The dotted region of Fig. 1 was kept inside the sorption-pumped vacuum can. The tip-sample separation (tunneling distance), for a fixed bias, was kept constant using a feedback loop. For a given set of tip and sample, the tip-sample distance determines the value of the tunnel current $I$ for a given bias. In our experiment, at each temperature the tunnel current $I \approx 1.5 \mathrm{nA}$ was first established at a bias $\approx 1.4 \mathrm{~V}$ for samples $\mathrm{A}$ and $\mathrm{B}$ and $2.5 \mathrm{~V}$ for sample $\mathrm{C}$. The stabilization voltages were chosen to be much larger than the expected gaps in the DOS for these materials, an estimate of which was initially made by trying different stabilization voltages. This was done to make sure that the tip did not crash into the sample, which can happen if the stabilization voltage is less than the gap which opens up in the DOS. The stabilization voltage for sample $\mathrm{C}$ was, therefore, kept larger because of the larger gap in the DOS of this material. The sample/hold circuit was in the sample mode during stabilization. After stabilizing the current, the feedback loop was put to hold mode so that the tunneling distance does not change when the bias is changed.

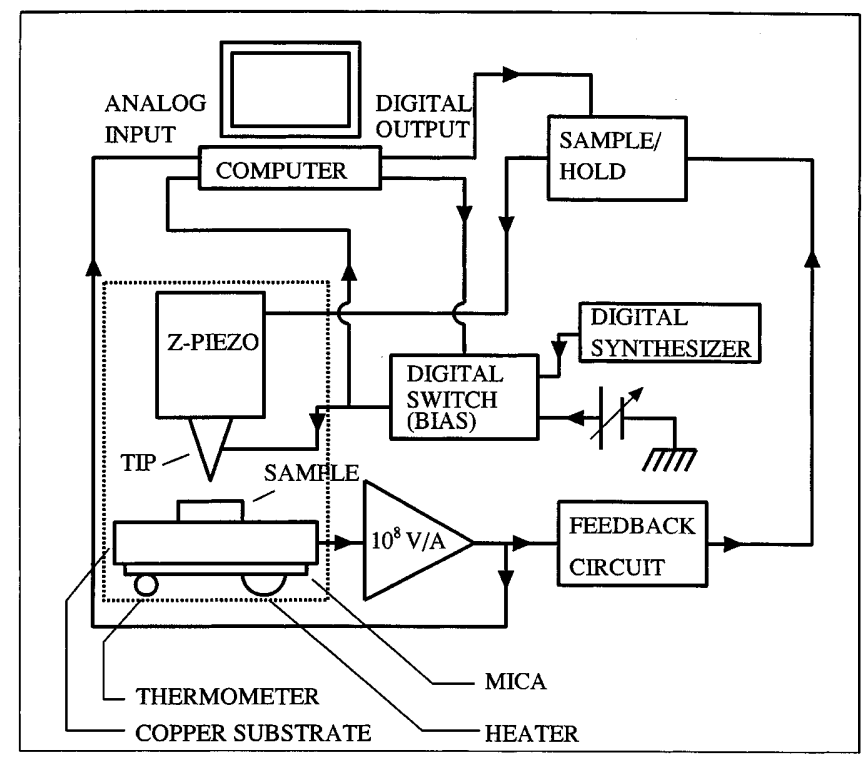

Fig. 1. Schematic diagram of the electronics of the STM setup used for the tunneling spectroscopy. The dotted region was in the temperature-controlled vacuum chamber
The bias was then swept across a certain range and a set of $I-V$ curves were recorded. It is from the stored curves that an average $I-V$ curve was obtained by taking an average of typically five curves. The time taken to record an $I-V$ curve is around $150 \mathrm{~ms}$. The dynamic conductance $(G=\mathrm{d} I / \mathrm{d} V)$ was obtained by taking the numerical derivative of the stored $I-V$ data. A typical $I-V$ curve contains 2000 points. (Note: fixing $I$ and $V$ at predetermined values ensures that the tunnel junctions at each temperature have similar characteristic parameters. This makes comparison of the tunneling data at different $T$ physically meaningful.) These measurements were done at ten or more points on the sample, within an area of $\approx 50 \mathrm{~nm} \times 50 \mathrm{~nm}$ at each temperature. We baked the sample to $400 \mathrm{~K}$ in situ in vacuum before starting the experiment. During cool-down, the temperature of the rest of the cryostat was lowered, first keeping the sample at high temperature to ensure that condensation of the residual gases occurs on the cryopumped sorption element and not on the sample surface. The gap values were estimated from the $G$ vs $V$ curves. The value of $2 \Delta_{\text {CO }}$ was taken as the difference of the two bias values (positive and negative) at which the value of $G$ goes below the noise level (which is $\approx 20 \mathrm{pA} / \mathrm{V}$ ). For a particular temperature the average of the gap values obtained at different points on the sample was taken as the mean gap value. The standard deviation was taken as the error bar. This error bar is thus a measure of the spatial variation of the gap value. It should be clarified here that, for the quantity $G_{0} / G_{0.5}$, the error bars denote the noise level near the zero bias and we have estimated this quantity only for samples A and B for which the spatial variation of the $I-V$ spectra is negligible.

\section{Results and discussion}

Figure 2 shows the resistivity data for all the samples. We can see from the figure that $\mathrm{La}_{0.8} \mathrm{Ca}_{0.2} \mathrm{MnO}_{3}$ shows an activated type of resistivity above $T_{\mathrm{C}} \approx 240 \mathrm{~K}$. The transport

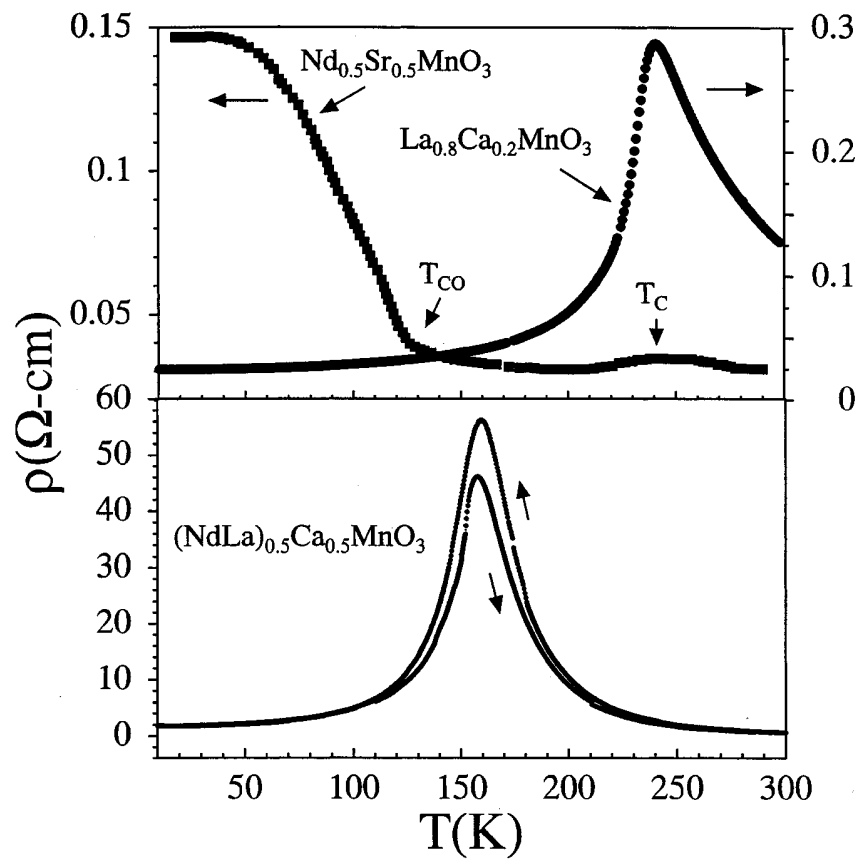

Fig. 2. The resistivity vs temperature for all the samples 
energy gap has been calculated from the high-temperature data to be around $0.12 \mathrm{eV}$. Below $T_{\mathrm{C}}$ the resistivity falls sharply. The resistivity data of $\mathrm{Nd}_{0.5} \mathrm{Sr}_{0.5} \mathrm{MnO}_{3}$ show that above $T_{\mathrm{C}} \approx 250 \mathrm{~K} \rho$ has a shallow dependence on $T$ and it is an activated semiconductor-like behavior albeit with a small gap. Below $250 \mathrm{~K}$ it has a metallic regime where resistivity decreases with temperature. Below $130 \mathrm{~K}$ there is a sharp rise in the resistivity. This is the $\mathrm{CO}$ temperature, $T_{\mathrm{CO}}$. The resistivity data of $(\mathrm{NdLa})_{0.5} \mathrm{Ca}_{0.5} \mathrm{MnO}_{3}$ above $158 \mathrm{~K}$ show an activated behavior. There is an increase in the activation gap (as estimated from the resistivity) at around $240 \mathrm{~K}$ where the sample becomes charge-ordered. The CO transition is clearer from the magnetic susceptibility data shown elsewhere [10]. The $\mathrm{CO}$ in $(\mathrm{NdLa})_{0.5} \mathrm{Ca}_{0.5} \mathrm{MnO}_{3}$ is distinct from the $\mathrm{CO}$ seen in $\mathrm{Nd}_{0.5} \mathrm{Sr}_{0.5} \mathrm{MnO}_{3}$ [10]. In $(\mathrm{NdLa})_{0.5} \mathrm{Ca}_{0.5} \mathrm{MnO}_{3}$ it is of incipient type and the $\mathrm{CO}$ state becomes unstable below $158 \mathrm{~K}$ where a ferromagnetic transition sets in and the resistivity drops sharply. There is a marked hysteresis in the heating and cooling curves. Also, the resistivity of $(\mathrm{NdLa})_{0.5} \mathrm{Ca}_{0.5} \mathrm{MnO}_{3}$ is much higher than both $\mathrm{Nd}_{0.5} \mathrm{Sr}_{0.5} \mathrm{MnO}_{3}$ and $\mathrm{La}_{0.8} \mathrm{Ca}_{0.2} \mathrm{MnO}_{3}$ even at room temperature. This is due to the fact that the $\left\langle r_{A}\right\rangle$ for $(\mathrm{NdLa})_{0.5} \mathrm{Ca}_{0.5} \mathrm{MnO}_{3}$ is $\approx 1.18 \AA$ which is smaller than for either of the other samples (which have $\left\langle r_{A}\right\rangle \geq 1.22 \AA$ ) [3].

In Fig. 3 we show representative $G(=\mathrm{d} I / \mathrm{d} V)-V$ curves for samples A and B as a function of $T$. The value of $T$ is rounded off to the nearest integer for simplicity (since the ranges of temperatures over which the changes occur are much larger). In sample $\mathrm{A}\left(\mathrm{La}_{0.8} \mathrm{Ca}_{0.2} \mathrm{MnO}_{3}\right)$ below the ferromagnetic $T_{\mathrm{C}}$ the $G-V$ curves are parabolic as expected in metal. In sample B it shows a metallic parabolic behavior in the temperature range $T_{\mathrm{C}}>T>T_{\mathrm{CO}}$. We extract the gap values from these $G-V$ graphs for the samples which show

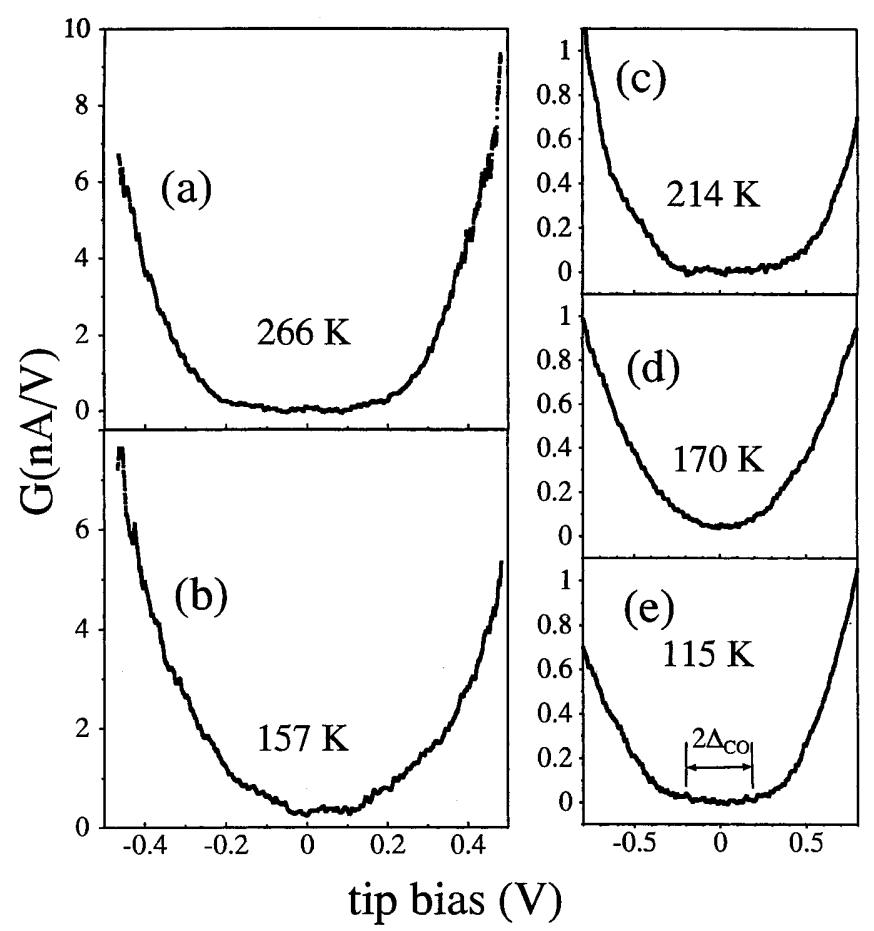

Fig. 3a-e. Representative $G(=\mathrm{d} I / \mathrm{d} V)$ vs $V$ curves at various temperatures showing the variation in the DOS. Curves a and $\mathbf{b}$ are for $\mathrm{La}_{0.8} \mathrm{Ca}_{0.8} \mathrm{MnO}_{3}$ and $\mathbf{c}, \mathbf{d}$ and $\mathbf{e}$ are for $\mathrm{Nd}_{0.5} \mathrm{Sr}_{0.5} \mathrm{MnO}_{3}$

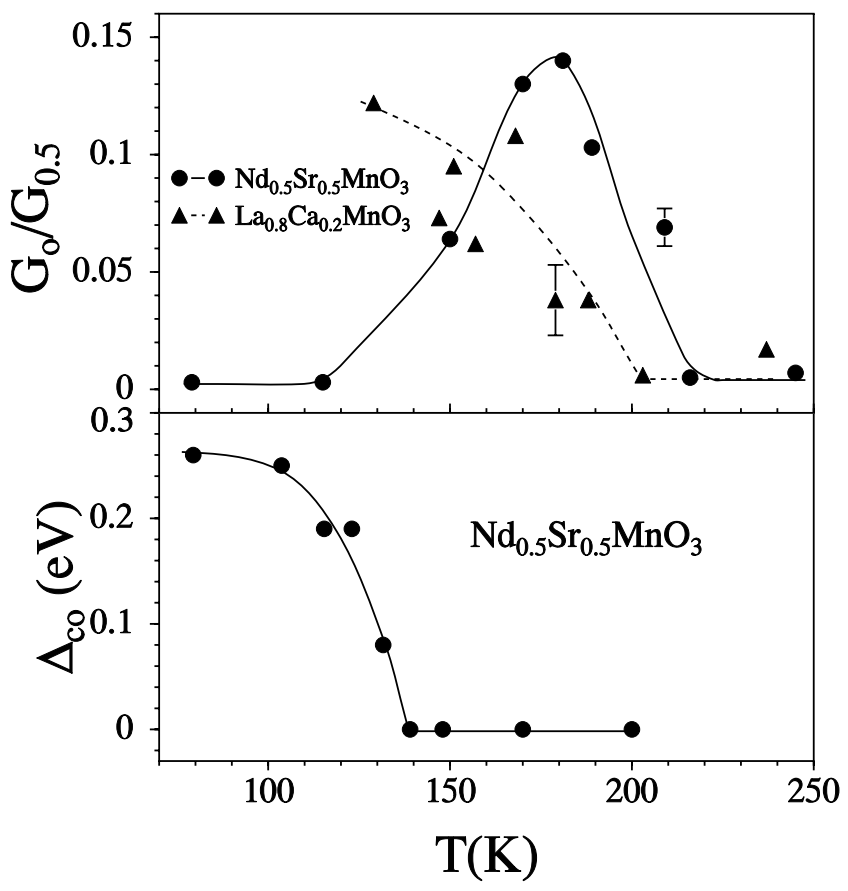

Fig. 4. The variation of the normalized conductance at zero bias, $G_{0} / G_{0.5}$, with temperature. The typical error in determining this quantity is shown for one point for each sample. The lower part shows the temperature dependence of the CO gap. The error bars are smaller than the symbol size

a gap at the Fermi level or get a relative measure of the DOS at the Fermi level from the normalized conductance ratio $\boldsymbol{g}=G_{0} / G_{0.5}$. The asymmetry in all the tunneling curves is noticeable and it also depends on the temperature.

Figure 4 shows the variation of the normalized zero bias conductance $\boldsymbol{g}$ (for $\mathrm{La}_{0.8} \mathrm{Ca}_{0.2} \mathrm{MnO}_{3}$ and $\mathrm{Nd}_{0.5} \mathrm{Sr}_{0.5} \mathrm{MnO}_{3}$ ). This ratio can be taken as a scaled measure of the value of DOS at $E_{\mathrm{F}}$. In both the materials $G_{0} / G_{0.5}$ increase rapidly as $T$ is reduced below the ferromagnetic transition temperature $T_{\mathrm{C}} . G_{0} / G_{0.5}$ starts rising above zero a few degrees below $T_{\mathrm{C}}$, for both samples. For the sample $\mathrm{La}_{0.8} \mathrm{Ca}_{0.2} \mathrm{MnO}_{3}$, which does not show $\mathrm{CO}$, the ratio reaches a finite limiting value as $T$ is lowered much below $T_{\mathrm{C}}$, indicating the existence of the metallic state down to low temperatures. In fact the DOS at $E_{\mathrm{F}}$ increases on cooling as the ferromagnetic state builds up in the material and it becomes more metallic. This is in conformity with photoemission experiments [13]. For the CO sample $\mathrm{Nd}_{0.5} \mathrm{Sr}_{0.5} \mathrm{MnO}_{3}$ the ratio $g$ shows a turn around below $175 \mathrm{~K}$ as $T \rightarrow T_{\mathrm{CO}}$. Eventually as the $\mathrm{CO}$ state forms below $T_{\mathrm{CO}}, \rho$ rises rapidly as $T$ is reduced and the ratio $g=0$. Below $140 \mathrm{~K}$ we see a measurable gap opening up as shown in the lower part of Fig. 4. The CO gap $\Delta_{\mathrm{CO}}$ reaches a limiting value of $\approx 0.27 \mathrm{eV}$ at $T<0.6 T_{\mathrm{CO}}$. The spatial variation of this value of the gap, at a particular temperature, is negligible, i.e. according to our definition, the error bars are smaller than the symbol size in Fig. 4.

In Fig. 5 we show the variation of $\Delta_{\mathrm{CO}}$ with temperature for $(\mathrm{NdLa})_{0.5} \mathrm{Ca}_{0.5} \mathrm{MnO}_{3}$. The gap increases continuously until around $160 \mathrm{~K}$ and then it falls sharply and goes to zero at around $120 \mathrm{~K}$. Also there is a region around $160 \mathrm{~K}$ (marked by the box) where the error bars are very large and also some of the gap values do not fall in the general trend. This implies that there is a spatial variation of the gap value around 


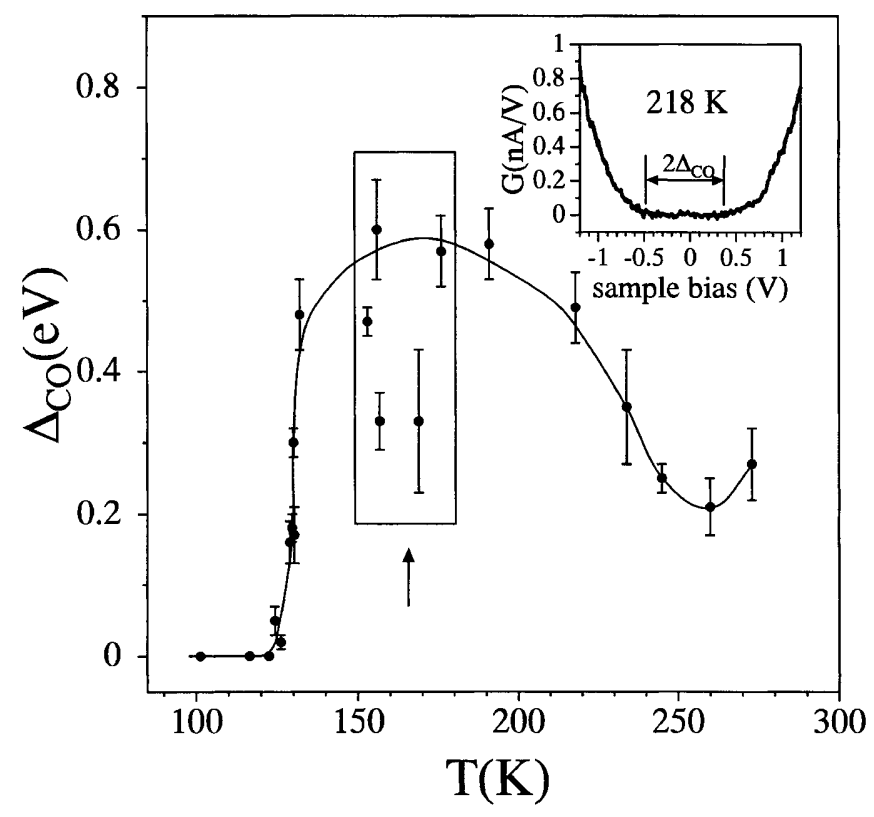

Fig. 5. Temperature variation of the $\mathrm{CO}$ gap of $(\mathrm{NdLa})_{0.5} \mathrm{Ca}_{0.5} \mathrm{MnO}_{3}$. The region marked with the box is a coexistence region for more than one phase. The inset shows the $G$ vs $V$ curve at $218 \mathrm{~K}$

this temperature. This is possible if there is a coexistence of more than one state, i.e. the charge-ordered insulating state $\left(\Delta_{\mathrm{CO}} \neq 0\right)$ and the ferromagnetic metallic states $\left(\Delta_{\mathrm{CO}}=0\right)$, at this temperature. This is also the reason why the resistivity data show hysteresis between the heating and cooling curves. The collapse of the CO gap in this material at low temperatures is an important observation and it has implications for the physics of the CO process in these materials [10].

From the above data it is clear that the DOS near the $E_{\mathrm{F}}$ and its variation with temperature is strongly dependent on $x$ and $\left\langle r_{A}\right\rangle$. The magnitude of the $\Delta_{\mathrm{CO}}$ gives us an estimate of the energies involved in the various interactions mentioned above. One likely way in which the $\mathrm{CO}$ takes place is through Coulomb repulsion $U_{\mathrm{nn}}$ of the charges sitting at nearest-neighbor sites. In that case $\Delta_{\mathrm{CO}}(T=0)$ should have values comparable to $U_{\mathrm{nn}}$. The exact value of $\Delta_{\mathrm{CO}}(T=0)$, however, depends on a number of details such as the bandwidth, strength of the lattice distortion, etc. Assuming absence of any screening in the charge-ordered state, we find $U_{\mathrm{nn}} \approx 0.38 \mathrm{eV}$ using a lattice constant of $0.38 \mathrm{~nm}$ for these solids and a dielectric constant of $\approx 10$. Thus $\Delta_{\mathrm{CO}}(T=0)$ as estimated from this experiment and $U_{\mathrm{nn}}$ are comparable.

To conclude, we have shown using temperature-dependent vacuum tunneling spectroscopy that the DOS of rare-earth manganates has a marked dependence on temperature. The values of the quantities such as the gap in the DOS at $E_{\mathrm{F}}$ and the normalized zero-bias conductance give us measures of the energies involved in the electronic processes in the different phases and the energies required for the transitions.

\section{References}

1. K. Chahara, T. Ohno, M. Kasai, Y. Kozono: Appl. Phys. Lett. 63, 1990 (1993)

2. R. Von Helmholt, J. Wecker, B. Holzapfel, L. Schutza, K. Samwer: Phys. Rev. Lett. 71, 2331 (1993)

3. H.Y. Hwang, S.-W. Cheong, P.G. Radaelli, M. Marezio, B. Batlogg: Phys. Rev. Lett. 75, 914 (1995)

4. R. Mahendiran, R. Mahesh, A.K. Raychaudhuri, C.N.R Rao: Phys. Rev. B 53, 12160 (1996)

5. H. Kuwahara, Y. Tomioka, A. Asamitsu, Y. Moritomo, Y. Tokura: Science 270, 961 (1995)

6. M.R. Lees, J. Barrett, G. Balakrishnan, D. McK. Paul, M. Yethiraj: Phys. Rev. B 52, R14 303 (1995)

7. T. Vogt, A.K. Cheetham, R. Mahendiran, A.K. Raychaudhuri, R. Mahesh, C.N.R. Rao: Phys. Rev. B 54, 15303 (1996)

8. K. Knizek, Z. Jirak, E. Pollert, F. Zounova, S. Vratislav: J. Solid State Chem. 100, 292 (1992)

9. R. Mahendiran, S.K. Tiwary, A.K. Raychaudhuri, T.V. Ramakrishnan, R. Mahesh, N. Rangavittal, C.N.R. Rao: Phys. Rev. B 53, 3348 (1996)

10. A. Arulraj, A. Biswas, A.K. Raychaudhuri, C.N.R. Rao, A.K. Cheetham, P. Woodward, T. Vogt, D. Cox: submitted for publication (1997)

11. A. Biswas, A.K. Raychaudhuri: J. Phys.: Condens. Matter (Lett.) 8, L739 (1996)

12. A. Biswas, A.K. Raychaudhuri, R. Mahendiran, R. Mahesh, C.N.R. Rao: J. Phys.: Condens. Matter (Lett.) 9, L355 (1997)

13. D.N. McIlroy, J. Zhang, S.-H. Liou, P.A. Dowben: Phys. Lett. A 207, 367 (1995) 